\title{
CARACTERÍSTICAS MORFOFISIOLÓGICAS ASSOCIADAS À QUALIDADE DE MUDAS DE Eucalyptus urophylla PRODUZIDAS EM DIFERENTES SUBSTRATOS ${ }^{1}$
}

\begin{abstract}
Orlando Amâncio de Oliveira Júnior ${ }^{2}$, Paulo Araquém Ramos Cairo ${ }^{3}$ e Adalberto Brito de Novaes ${ }^{3}$
RESUMO - Neste estudo foram avaliados os efeitos de diferentes combinações de substratos sobre características morfológicas e fisiológicas relacionadas à qualidade de mudas de Eucalyptus urophylla. O experimento foi conduzido em um viveiro florestal a pleno sol, no qual foram produzidas mudas em tubetes de 54 cc e irrigadas por microaspersão. Empregou-se o delineamento experimental inteiramente casualizado com 12 tratamentos e quatro repetições. Os tratamentos foram baseados nas seguintes combinações de substratos: dois substratos comerciais (Vivatto Slim Plus e Mecplant), esterco bovino, pó de casca de coco, vermiculita e uma combinação utilizada por uma empresa do ramo florestal. Em todos os tratamentos foi adicionado Osmocote, fertilizante mineral de suprimento controlado de NPK. Foram avaliadas as seguintes características: Índice de Velocidade de Emergência (IVE), Emergência Total de Plântulas (EMER), Índice de Qualidade Dickson (IQD), Índice SPAD e Potencial de Regeneração de Raízes (PRR). Em geral, os melhores resultados foram obtidos nos tratamentos que continham esterco bovino e pó de casca de coco nas combinações de substratos. Para a qualificação das mudas, os valores de IQD mostraram-se, quase sempre, relacionados positivamente aos de PRR, exceto o substrato 100\% da Vivatto Slim Plus, em que os maiores valores de IQD estiveram associados a valores mais baixos de PRR. A combinação de $40 \%$ de esterco bovino, $20 \%$ de pó de casca de coco e $40 \%$ de vermiculita resultou nas maiores médias do índice SPAD. A combinação de $70 \%$ de esterco bovino e 30\% de pó de casca de coco proporcionou a produção de mudas com os maiores valores de PRR, as quais apresentaram qualidade superior à das demais combinações de substratos, com base nas características avaliadas.
\end{abstract}

Palavras-chave: Eucalipto, Qualidade de mudas e Características fisiológicas.

\section{MORPHOPHYSIOLOGICAL CHARACTERISTICS ASSOCIATED TO QUALITY OF Eucalyptus urophylla SEEDLINGS PRODUCED ON DIFFERENT SUBSTRATES}

\begin{abstract}
It was evaluated in this study the effects of different combinations of substrates on the morphologic and physiological characteristcs related to the quality of Eucalyptus urophylla seedlings. The experiment was conducted in a a forest nursery on the sun, in which seedlings were produced in 54 cc tubes and irrigated by micron aspersion. It was used a complete random experimental design with 12 treatments and four replicates. Treatments were based on the following combinations of substrate: two commercial substrates (Vivatto Slim Plus and Mecplant), manure, coconut bark powder, vermiculite, and a mixture substrate used by a Forest Company. Osmocote, a mineral fertilizer which has a controlled NPK supply, was added into all treatments. The following characteristics were evaluated: root regeneration potential (RRP); total seedling growth (TSG); growth speed index (GSI), Dickson quality index (IQD), and SPAD index. Overall, the best results were obtained in treatments with coconut bark powder and manure. Concerning seedling quality, IQD values were almost always positively associated to RRP, except the 100\% Vivatto Slim Plus substrates which showed lower RRP. The highest means of SPAD indexes were obtained from the combination of $40 \%$ manure, 20\% coconut bark, and $40 \%$ vermiculite. The combination of $70 \%$ manure and $30 \%$ coconut bark powder provided seedlings production with the highest values of RRP, which presented superior quality than the other substrates combination, based on the evaluated characteristics.
\end{abstract}

Keywords: Eucalyptus, Seedling quality and Physiological characteristics.

\footnotetext{
${ }^{1}$ Recebido em 23.09.2009 e aceito para publicação em 31.10.2011.

${ }^{2}$ Mestre em Agronomia, Universidade Estadual do Sudoeste da Bahia, UESB, Brasil. E-mail: <orlando.amancio@yahoo.com.br>. ${ }^{3}$ Departamento de Fitotecnia e Zootecnia, Universidade Estadual do Sudoeste da Bahia, UESB, Brasil. E-mail: <pcairo@uol.com.br> e <adalberto.brito@globo.com>.
} 


\section{INTRODUÇÃO}

Na região Sudoeste da Bahia, particularmente no Planalto de Conquista, a atividade de reflorestamento desponta como uma das alternativas socioeconômicas mais promissoras para atender às demandas da indústria madeireira regional e, ao mesmo tempo, contornar os problemas de desmatamento desenfreado. Todavia, a implantação de programas florestais com povoamentos mais produtivos requer novas tecnologias, que permitam suportar as adversidades do meio, apresentar altos percentuais de sobrevivência no campo, possibilitar a diminuição da frequência dos tratos culturais do povoamento recém-implantado e produzir árvores com volume e qualidades desejáveis (NOVAES, 1998; GOMES, 2001).

O sucesso na implantação de programas florestais requer esforço permanente na produção de mudas com qualidade cada vez maior. A obtenção de mudas em curto prazo, com alto vigor e baixo custo de produção, depende diretamente de fatores como tipos de substrato, recipiente e fertilizante utilizados; qualidade do material genético reprodutivo; e manejo da irrigação (STURION eANTUNES, 2000; BERNARDINO et al., 2005; DAVIDE; FARIA, 2008).

Com a expansão das fronteiras agrícolas e dos plantios florestais, torna-se crescente a demanda de substratos para a produção de mudas. O uso de substratos está relacionado ao cultivo fora do solo, realizado muitas vezes em ambiente protegido e que emprega, em geral, volume limitado contido em recipientes (KÄMPF, 2001). Diversos materiais orgânicos e inorgânicos têm sido utilizados para a confecção de substratos, havendo a necessidade de se determinarem os mais apropriados para cada espécie. Considerando que cada substrato apresenta peculiaridades em relação às suas características químicas e físicas, acredita-se que o uso de diferentes substratos, ou até mesmo as diversas combinações entre eles, pode constituir-se num fator capaz de influir no padrão de qualidade de mudas de eucalipto. Além disso, dificilmente um material isolado apresenta características ótimas para o crescimento de mudas. Para a formação de mudas de alto padrão de qualidade, é essencial que se desenvolvam estudos sobre substratos capazes de disponibilizar, de maneira equilibrada, nutrientes e umidade e que possuam características físicas que favoreçam o crescimento, sem restrições e deformações das raízes (CARNEIRO, 1995; CUNHA et al., 2005; LIMA et al., 2006; WENDLING et al., 2006).

Revista Árvore, Viçosa-MG, v.35, n.6, p.1173-1180, 2011

Revista Árvore, Viçosa-MG, v.35, n.6, p.1173-1180, 2011
Os substratos comerciais utilizados na produção de mudas de espécies florestais no Sudoeste baiano são fabricados nas Regiões Sul e Sudeste do país. A fim de diminuir o custo da produção de mudas, é conveniente que se façam estudos comparativos entre mudas obtidas com esses substratos e aquelas com substratos abundantes na região onde as mudas serão produzidas, desde que sejam preservados os padrões de qualidade. A necessidade desses estudos é evidente quando se observa a escassez de informações sobre a qualidade dos substratos locais, que são de custo mais baixo que os substratos oriundos de outras regiões.

Tendo em vista a importância de se conhecer melhor o potencial de utilização de alguns substratos mais viáveis e facilmente encontrados no Sudoeste baiano, bem como diminuir a dependência e os custos que envolvem a produção de mudas que utilizam substratos oriundos de outras regiões, neste trabalho se propôs avaliar os efeitos de diferentes combinações de substratos sobre a qualidade de mudas de Eucalyptus urophylla produzidas em tubetes, em Vitória da Conquista, Bahia.

\section{MATERIAL E MÉTODOS}

O trabalho foi desenvolvido em duas etapas, ambas em Vitória da Conquista: uma no Viveiro da Empresa Florestal Consultoria LTDA., onde as mudas foram produzidas; e a outra na Universidade Estadual do Sudoeste da Bahia (UESB), para onde as mudas foram levadas para as avaliações subsequentes.

A semeadura foi realizada em 1\%10/2008, utilizando-se sementes de Eucalyptus urophylla obtidas de árvoresmatriz em Área de Produção de Sementes. O ciclo de produção das mudas correspondeu a 100 dias após a semeadura.

Os substratos utilizados para a produção das mudas continham diferentes combinações de materiais orgânicos e inorgânicos. O esterco bovino (EB) foi coletado na área experimental da UESB, sendo curtido e passado em peneira de 4,76 mm. O pó de casca de coco (PC), constituído de pó e fibras da casca do coco, foi adquirido no comércio local. Os dois substratos anteriores (EB e PC) foram submetidos à análise química, descrita na Tabela 1. O substrato comercial Vivatto Slim Plus (VI), da empresa Technes, constituído à base de casca de pinus bioestabilizada e com a presença de vermiculita, 
também foi adquirido no comércio local. A vermiculita (VE) utilizada foi a fina expandida. Outro substrato comercial empregado foi o Mecplant (PI) (casca de pinus bioestabilizada), produzido pela empresa Mecprec. Por último, utilizou-se uma mistura de substratos (50\% de casca de arroz carbonizada, 30\% de Mecplant e 20\% de vermiculita), que é um formulado utilizado por uma empresa do ramo florestal (SU) e obtido por meio de doação da Empresa Suzano Papel e Celulose, situada na cidade de Teixeira de Freitas, BA.

A formulação dos tratamentos foi elaborada por meio da mistura manual dos substratos, obtendo-se 12 tratamentos, a partir dos diferentes componentes selecionados (Tabela 2). Para a padronização da fertilização, utilizou-se adubação com Osmocote, adubo mineral de liberação controlada, à base de NPK (06-19-06), de modo que todos os tratamentos atingissem 2,5 g/litro de substrato.

Adotou-se o delineamento experimental inteiramente ao acaso, com 12 tratamentos (Tabela 2) e quatro repetições, em que cada repetição consistiu de 40 mudas. Os resultados foram submetidos à análise de variância, empregando-se o teste $\mathrm{F}$ a $5 \%$. As médias foram comparadas pelo teste de Duncan a 5\%. Em todas as análises de variância, os resultados foram submetidos aos testes de normalidade e homogeneidade, não havendo a necessidade de transformação dos dados.

Os recipientes utilizados na semeadura foram tubetes de polipropileno, com volume de $54 \mathrm{cc}$, altura de $12,5 \mathrm{~cm}$, $3 \mathrm{~cm}$ de diâmetro superior e $1 \mathrm{~cm}$ de diâmetro na parte inferior. Como suportes para os tubetes, foram utilizadas bandejas de polipropileno com capacidade para 140 tubetes, as quais foram colocadas diretamente sobre solo plano. A semeadura foi feita manualmente, adicionando-se em torno de quatro sementes por recipiente. Em seguida, procedeu-se à cobertura das sementes, com uma camada constituída da mesma mistura usada para o enchimento dos tubetes. O raleamento foi efetuado quando as plântulas apresentaram aproximadamente $5 \mathrm{~cm}$ de altura, preservando-se apenas a de melhor formação e mais central, em cada recipiente, conforme Sturion et al. (2000). A irrigação foi efetuada diariamente, mediante o sistema de microaspersão, cinco vezes por dia, num intervalo de $3 \mathrm{~h}$, com início às 6 h e término às 18 h (REIS et al., 2008).

Para a avaliação do índice de velocidade de emergência (IVE) e da emergência total de plântulas (EMER), foi feita a contagem diária das plântulas que emergiram até o momento de sua estabilização e a emergência total nas primeiras repetições, iniciando-se as verificações no oitavo dia após a semeadura e encerrando-as no $15^{\circ}$ dia. O critério adotado como referência para determinar se as plântulas tinham emergido foi o início visível do aparecimento das folhas cotiledonares. Ao final do período, calculou-se a porcentagem total de emergência (EMER) de cada unidade experimental, bem como o índice de velocidade de emergência (IVE), segundo Maguire (1962), pela fórmula: $\mathrm{IVE}=\mathrm{E} 1 / \mathrm{D} 1+\mathrm{E} 2 / \mathrm{D} 2+\ldots \ldots . \mathrm{En} / \mathrm{Dn}(\mathrm{E} 1=$ número de plântulas que emergiram e D1 = número de dias após a semeadura).

Tabela 1 - Análise do esterco bovino (EB) e do pó de casca de coco (PC), utilizados como substratos para a produção de mudas de Eucalyptus urophylla.

Table 1 - Analysis of the cattle manure (CM) and the coconut bark powder (PC), used as substrates to Eucalyptus urophylla seedlings production.

\begin{tabular}{|c|c|c|c|}
\hline \multirow[t]{2}{*}{ Características } & \multirow[t]{2}{*}{ Unidades } & \multicolumn{2}{|c|}{ Substratos } \\
\hline & & EB & PC \\
\hline CE & $\mathrm{mS} \mathrm{cm}^{-1}$ & 2,90 & 1,63 \\
\hline $\mathrm{pH}$ & $\mathrm{H}_{2} \mathrm{O}$ & 7,50 & 7,80 \\
\hline $\mathrm{P}$ & $\mathrm{mg} \mathrm{dm}-3$ & 36,00 & 3,50 \\
\hline $\mathrm{K}^{+}$ & mmolc dm ${ }^{-3}$ & 29,70 & 14,30 \\
\hline $\mathrm{Na}^{+}$ & mmolc dm ${ }^{-3}$ & 5,60 & 2,90 \\
\hline $\mathrm{Ca}^{++}$ & mmolc dm ${ }^{-3}$ & 2,50 & 3,00 \\
\hline $\mathrm{Mg}^{++}$ & mmolc dm ${ }^{-3}$ & 3,50 & 3,00 \\
\hline $\mathrm{Cu}$ & $\mathrm{mg} \mathrm{dm} \mathrm{m}^{-3}$ & 0,36 & 0,06 \\
\hline Mn & $\mathrm{mg} \mathrm{dm}^{-3}$ & 1,20 & 0,05 \\
\hline $\mathrm{Zn}$ & $\mathrm{mg} \mathrm{dm} \mathrm{d}^{-3}$ & 0,20 & 1,00 \\
\hline $\mathrm{Fe}$ & $\mathrm{mg} \mathrm{dm}^{-3}$ & 5,00 & 3,00 \\
\hline
\end{tabular}

* Extrator de Mehlich-1. Utilizou-se o método 1:2 (v/v) de substrato para água na extração e determinação das características. 
Tabela 2 - Porcentagem dos materiais utilizados na formulação das combinações de substratos (tratamentos). (volume/volume). Table 2 - Percentage of materials used for preparation of compounds of substrates (treatments). (volume/volume).

\begin{tabular}{|c|c|c|c|c|c|c|}
\hline Tratamento & VI & $\mathrm{SU}$ & P I & EB & PC & $\mathrm{VE}$ \\
\hline T1 & 100 & - & - & - & - & - \\
\hline Т 2 & 70 & - & - & 30 & - & - \\
\hline Т 3 & 50 & - & - & 30 & 20 & - \\
\hline $\mathrm{T} 4$ & - & 100 & - & - & - & - \\
\hline T 5 & - & 70 & - & 30 & - & - \\
\hline T 6 & - & 50 & - & 30 & 20 & - \\
\hline $\mathrm{T} 7$ & - & - & 70 & 30 & - & - \\
\hline Т 8 & - & - & 50 & - & 20 & 30 \\
\hline Т 9 & - & - & - & 70 & 30 & - \\
\hline $\mathrm{T} 10$ & - & - & - & 50 & 20 & 30 \\
\hline $\mathrm{T} 11$ & - & - & - & 33,3 & 33,3 & 33,3 \\
\hline $\mathrm{T} 12$ & - & - & - & 40 & 20 & 40 \\
\hline
\end{tabular}

$\mathrm{VI}=$ substrato comercial Vivatto Plus; $\mathrm{SU}=50 \%$ de casca de arroz carbonizada $+30 \%$ de Mecplant $+20 \%$ de vermiculita; $\mathrm{PI}=$ Mecplant, substrato comercial à base de casca de pinus bioestabilizada; $\mathrm{EB}=$ esterco bovino curtido; PC= pó de casca de coco; e VE = vermiculita fina.

Aos 100 dias após a semeadura, as mudas foram retiradas do viveiro e cinco mudas por repetição foram coletadas aleatoriamente, totalizando 20 mudas/ tratamento, para verificação de alguns parâmetros fitotécnicos. A altura $(\mathrm{H})$ das plantas foi verificada por medição com régua graduada, tomando-se como padrão a gema terminal (meristema apical). Na medição do diâmetro (D), tomou-se como referência o colo da muda, o qual foi medido com paquímetro digital. Para a obtenção do peso da matéria seca da parte aérea (PSA) e do sistema radicial (PSR), essas partes foram acondicionadas separadamente em embalagens de papel, por $48 \mathrm{~h}$, em estufa de circulação forçada de ar, à temperatura de $65^{\circ} \mathrm{C} \pm 3^{\circ} \mathrm{C}$, com as embalagens abertas, para facilitar a perda de umidade (MORAES NETO et al., 2003). As pesagens dos materiais foram realizadas em balança digital de precisão, sendo o peso da matéria seca total (PST) resultante da soma de PSA com PSR. Esses parâmetros fitotécnicos foram necessários para a obtenção do índice de qualidade Dickson, calculado com base na seguinte fórmula (DICKSON et al., 1960):

$$
\mathrm{IQD}=\frac{\operatorname{PST}(\mathrm{g})}{\mathrm{H}(\mathrm{cm}) / \mathrm{D}(\mathrm{mm})+\operatorname{PSA}(\mathrm{g}) / \operatorname{PSR}(\mathrm{g})}
$$

A leitura do índice relativo de clorofila (SPAD) foi determinada com um medidor portátil, modelo SPAD502 (Soil and Plant Analysis Development), da Minolta, avaliando-se as mudas em um único período, ao final do seu ciclo de 100 dias. O índice médio de cada tratamento foi resultante da avaliação de cinco folhas por repetição, em mudas distintas, sendo selecionadas folhas completamente expandidas, situadas no terço médio superior das mudas, com aferições na extremidade de cada folha, adotando-se a unidade SPAD.

Para avaliação do potencial de regeneração de raízes (PRR), foram selecionadas e retiradas oito mudas do viveiro, para compor cada tratamento. As mudas foram submetidas a uma lavagem cuidadosa e à poda das raízes laterais, a uma distância de $4 \mathrm{~cm}$ do eixo da raiz pivotante, que também foi podada a $15 \mathrm{~cm}$ do colo, visando à determinação do número total de raízes e à distribuição de extremidades de raízes visíveis nas paredes dos tubos das mudas transplantadas (NOVAES et al., 2002).

Os recipientes utilizados para avaliação do $\mathrm{PRR}$ foram tubos plásticos transparentes, confeccionados a partir de garrafas plásticas de refrigerante (2 L) que, após a remoção dos gargalos, tomaram a forma de tubos, com dimensões de $25 \mathrm{~cm}$ de altura e $31 \mathrm{~cm}$ de circunferência, com volume de 1,9 L. Para ocorrer a drenagem do excesso de água, evitando o encharcamento, realizou-se a perfuração do fundo de cada recipiente, sendo os tubos preenchidos com o substrato comercial Vivatto Slim Plus. Foram utilizados 96 tubos, sendo oito para cada um dos 12 tratamentos. Após o preparo das mudas, com o sistema radicial devidamente podado, elas foram transplantadas para os respectivos recipientes. Em seguida, os tubos foram revestidos por lona plástica de cor preta, para evitar a incidência de luz. As regas foram realizadas diariamente,

Revista Árvore, Viçosa-MG, v.35, n.6, p.1173-1180, 2011 
de forma homogênea, em todos os tratamentos. As leituras foram efetuadas na parte lateral dos tubos, em intervalos de dois dias, num total de 15 avaliações, demarcando os pontos tocados por raízes que apresentassem comprimento a partir de $1 \mathrm{~cm}$. Ao final do experimento foram somados todos os valores correspondentes aos pontos de raízes de cada planta, chegando-se ao PRR.

\section{RESULTADOS}

Os resultados apresentados na Figura 1 indicaram valores de IVE estatisticamente iguais para a maioria dos tratamentos, exceto para T1, T2 e T3 (à base de Vivatto Slim Plus) e T11 (1EB:1PC:1VE), que promoveram valores inferiores. As combinações de substrato à base de Vivatto Slim Plus (T1, T2 e T3) também influíram nas
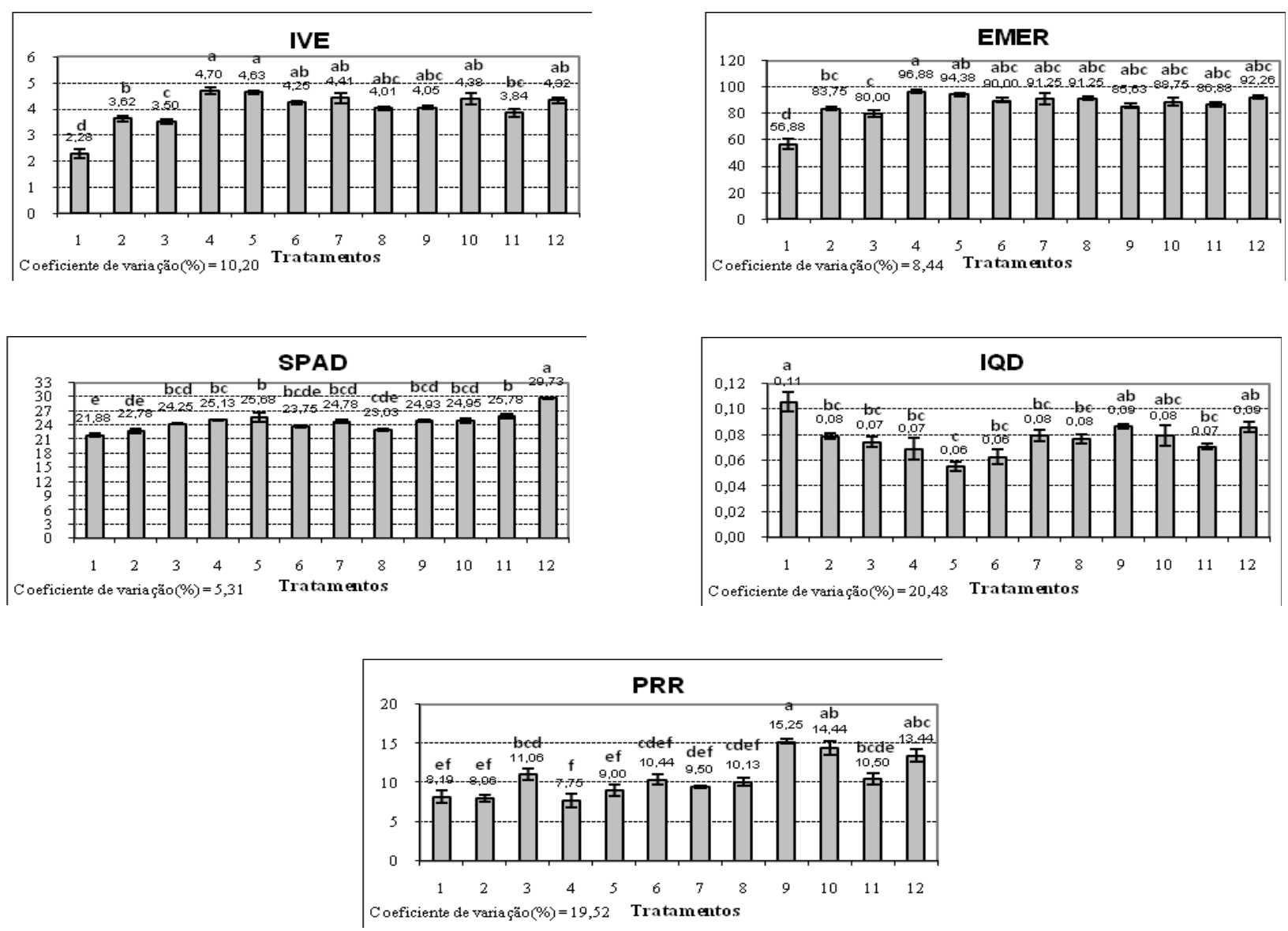

\begin{tabular}{|c|c|c|c|}
\hline$T 1=(100 V I)$ & $T 4=(100 S U)$ & T7 = (70PI + 30EB) & $\mathrm{T} 10=(50 \mathrm{~EB}+20 \mathrm{PC}+30 \mathrm{VE})$ \\
\hline $\mathrm{T} 2=(70 \mathrm{VI}+30 \mathrm{~EB})$ & T5 $=(70 \mathrm{SU}+30 \mathrm{~EB})$ & $\mathrm{T} 8=(50 \mathrm{PI}+20 \mathrm{PC}+30 \mathrm{VE})$ & $\mathrm{T} 11=(33 \mathrm{~EB}+33 \mathrm{PC}+33 \mathrm{VE})$ \\
\hline $\mathrm{T3}=(50 \mathrm{VI}+30 \mathrm{~EB}+20 \mathrm{PC})$ & $\mathrm{T} 6=(50 \mathrm{SU}+30 \mathrm{~EB}+20 \mathrm{PC})$ & $\mathrm{T} 9=(70 \mathrm{~EB}+30 \mathrm{PC})$ & $\mathrm{T} 12=(40 \mathrm{~EB}+20 \mathrm{PC}+40 \mathrm{VE})$ \\
\hline
\end{tabular}

Figura 1 - Valores médios do índice de velocidade de emergência (IVE), da emergência total de plântulas (EMER), do Índice SPAD, do Índice de Qualidade Dickson (IQD) e do Potencial de Regeneração de Raízes (PRR) das mudas de Eucalyptus urophylla.

Figure 1 - Means values of growth velocity index (GVI), seedling total emergency (STE), SPAD index, Dickson quality index (IQD) and root regeneration potential (RRP) of Eucalyptus urophylla seedlings. 
menores porcentagens de emergência total das plântulas (EMER). OT1 (100\% Vivatto Slim Plus) registrou o menor valor de EMER (56,88\%), enquanto o T4 (composto da empresa florestal) apresentou quase a totalidade das plântulas emergidas (96,88\%). Analisando os dados de EMER, com exceção dos tratamentos T1, T2 e T3, os demais tiveram resultados similares. As variações dos efeitos dos tratamentos sobre a emergência (IVE e EMER) das mudas revelaram comportamentos relativamente semelhantes, em ambas as características avaliadas.

O Índice de Qualidade Dickson (IQD) registrou os maiores valores na mistura de esterco bovino, pó de casca de coco e vermiculita (T9, T10 e T12). O substrato comercial Vivatto Slim Plus (T1) também proporcionou às mudas elevado IQD. Os tratamentos com menor IQD foram os que apresentaram, em suas combinações, a casca de arroz carbonizada (T4, T5 e T6), que é utilizada no formulado da empresa florestal.

Em relação ao índice SPAD, o T12 (4EB:2PC:4VE) destacou-se dos demais, o que sugere a presença de maior teor de clorofila e nitrogênio nas suas folhas. O substrato comercial Vivatto Slim Plus, por sua vez, promoveu os menores valores do índice SPAD.

A combinação de esterco bovino, pó de casca de coco e vermiculita influenciou positivamente na formação de novas raízes, proporcionando os maiores valores de potencial de regeneração de raízes (PRR). Os valores de PRR das mudas produzidas pelas combinações de substrato em T9 e T10 - respectivamente 15,59 e 14,44 - foram superiores ao dobro do valor encontrado no tratamento com menor número de raízes regeneradas, o T4 $(7,03)$. Os tratamentos T9 e T10 promoveram a formação de mudas com os maiores valores de altura da parte aérea (H) e pesos da matéria seca total (PST), colaborando para o maior PRR.

\section{DISCUSSÃO}

O melhor desenvolvimento radicular e vegetativo pode ser obtido a partir da maior rapidez de estabelecimento, ou seja, maior IVE (SOARES et al., 2008). Isso, provavelmente, deve ocorrer em função do antecipado estabelecimento da parte aérea, que permite a antecipada captação dos raios solares, com a realização da fotossíntese, desencadeando os processos fisioquímicos da planta (TAIZ; ZEIGER, 2009). Também, Martins et al. (1999) afirmaram que plântulas que emergem mais lentamente e passam mais tempo nos estádios iniciais de crescimento podem tornar-se mais vulneráveis às condições adversas do meio.

A padronização na produção das mudas, desde a emergência, favorece a uniformização no seu desenvolvimento, o que possibilita a saída de mudaspadrão do viveiro para o campo, admitindo-se em iguais condições de desempenho após o plantio.

O Índice de Qualidade Dickson (IQD) permite classificar mudas quanto à qualidade a partir das relações de parâmetros morfológicos, em que, quanto maior o índice, melhor qualidade terá a muda avaliada (GOMES, 2001). Bernardino et al. (2005), avaliando a qualidade de mudas de Anadenanthera macrocarpa (Benth.) Brenan, também afirmaram que as mudas com maior IQD são classificadas como de melhor qualidade. Os menores valores do IQD das mudas dos tratamentos T4, T5 e T6 podem ter sido influenciados pela má agregação física e possível limitação de nutrientes e pouca retenção de água, na mistura de casca de arroz carbonizada com pó de coco (WEBER et al., 2003).

O T12, tratamento com o maior número de folhas, apresentou também maior SPAD. Esse resultado pode estar relacionado ao fato de que o nitrogênio, por ser nutriente bastante móvel na planta, é direcionado rapidamente para as folhas mais jovens (SENA JÚNIOR et al., 2008). Apesar de maior SPAD e número de folhas, esses parâmetros não foram suficientes para a avaliação da qualidade das mudas deste trabalho. Já para Reis et al. (2006) e Godoy et al. (2008), avaliando cafeeiro, a leitura SPAD de clorofila correlacionou-se positivamente com o teor de $\mathrm{N}$ nas folhas e com a produtividade.

Neste trabalho, a análise do teor de clorofila e nitrogênio, pelo SPAD, não permitiu conclusões sobre o peso de matéria seca da parte aérea (PSA), em que as mudas com os maiores SPAD não foram as que obtiveram maiores PSA.

Barroso et al. (2000), avaliando mudas de eucalipto, assim como Bonfim (2007), analisando qualidade de mudas de madeira-nova, encontraram valores do potencial de regeneração de raízes (PRR) próximos aos deste trabalho. Um substrato que se destaca no suporte às mudas, favorecendo o maior potencial de regeneração radicial, provavelmente favorecerá o estabelecimento das mudas no campo com maior eficiência e maior vigor, no qual as raízes explorarão a maior área do solo, em

Revista Árvore, Viçosa-MG, v.35, n.6, p.1173-1180, 2011

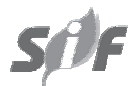


busca de água e nutrientes, proporcionando superior crescimento inicial no campo (BARROSO et al., 2000; LOPES, 2005; BONFIM, 2007).

Novaes etal.(2002), avaliando Pinnus taeda, constataram que as mudas que apresentaram maior capacidade de regeneração de suas raízes também tiveram melhor desempenho inicial após o plantio. Barroso et al. (2000) também constataram que o tratamento que apresentou menor percentual de sobrevivência no campo foi o que registrou menores índices de regeneração de raízes (PRR).

Com relação ao PRR, deduz-se também que o maior peso de matéria fresca total possibilita um bom suporte aéreo, que provavelmente irá colaborar para a produção de fotoassimilados e posterior regeneração das raízes, possibilitando maior PRR.

A presença do esterco bovino nas combinações de substratos resultou em alguns benefícios, a exemplo do maior fornecimento de nutrientes e da diminuição de custos na produção de mudas, o que pode amenizar a dependência da atividade florestal com relação aos substratos comerciais.

\section{CONCLUSÕES}

- Com base nas características avaliadas, as maiores médias foram obtidas nos tratamentos que utilizaram o esterco bovino e o pó de casca de coco nas combinações dos substratos.

- Para qualificação das mudas, os valores de IQD mostraram-se, quase sempre, relacionados positivamente aos de PRR, exceto com ao substrato $100 \%$ da Vivatto Slim Plus, em que os maiores valores de IQD estiveram associados a valores mais baixos de PRR.

- A combinação de $40 \%$ de esterco bovino, $20 \%$ de pó de casca de coco e $40 \%$ de vermiculita resultou nas maiores médias do índice SPAD.

A combinação de $70 \%$ de esterco bovino e 30\% de pó de casca de coco na formulação de substratos proporcionou a produção de mudas com maior potencial de regeneração de raízes (PRR), sendo considerado o melhor tratamento.

\section{REFERÊNCIAS}

BARROSO, D. G. et al. Regeneração de raízes de mudas de eucalipto em recipientes e substratos. Scientia Agricola, v.57, n.2, p.229-237, 2000.
BERNARDINO, D. C. S. et al. Crescimento e qualidade de mudas de Anadenanthera macrocarpa (Benth.) brenan em resposta à saturação por bases do substrato. Revista Árvore, v.29, n.6, p.863-870, 2005.

BONFIM, A. A. Qualidade de mudas de madeira-nova (Pterogyne nitens Tull.) produzidas em tubetes e sacolas plásticas e seu desempenho no campo. 2007. 70f. Dissertação (Mestrado em Agronomia) Universidade Estadual do Sudoeste da Bahia, Vitória da Conquista, 2007.

CARneiro, J. G. A. Produção e controle de qualidade de mudas florestais. Curitiba: UFPR/FUPEF, 1995. 451p.

CUNHA, A. O. et al. Efeitos de substratos e das dimensões dos recipientes na qualidade das mudas de Tabebuia impetiginosa (Mart. Ex D.C.) Standl. Revista Árvore, v.29, n.4, p.507-516, 2005.

DAVIDE, A. C.; FARIA, J. M. R. Produção de sementes e mudas de espécies florestais. In: DAVIDE, A. C.; SILVA, E. A. A. (Eds) Produção de sementes e mudas de espécies florestais. Lavras: Universidade Federal de Lavras, 2008. 175p.

DICKSON, A.; LEAF, A. L.; HOSNER, J. F. Quality appraisal of white spruce and white pine seedling stock in nurseries. Forest Chronicle, v.36, n.1, p.10-13, 1960.

GODOY, L. J. G. et al. Índice relativo de clorofila e o estado nutricional em nitrogênio durante o ciclo do cafeeiro fertirrigado. Revista Brasileira de Ciência do Solo, v.32, n.1, p.217-226, 2008.

GOMES, J. M. Parâmetros morfológicos na avaliação da qualidade de mudas de Eucalyptus grandis, produzidas em diferentes tamanhos de tubete e de dosagens de N-P-K. 2001. 166f. Tese (Doutorado em Ciência Florestal) - Universidade Federal de Viçosa, Viçosa, MG, 2001.

KÄMPF, A. N. Substratos para plantas: um desafio para a ciência do solo. Revista Opinião, v.26, n.1, p.5-16, 2001.

LIMA, R. L. S. et al. Volume de recipientes e composição de substratos para produção de mudas de mamoneira. Ciência e

Agrotecnologia, v.30, n.3, p.480-486, 2006.

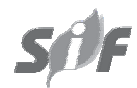

Revista Árvore, Viçosa-MG, v.35, n.6, p.1173-1180, 2011 
LOPES, E. D. Qualidade de mudas de Eucalyptus urophylla, E. camaldulensis e Corymbia citriodora produzidas em blocos prensados e em dois modelos de tubetes e seu desempenho no campo. 2005. 82f. Dissertação (Mestrado em Agronomia) Universidade Estadual do Sudoeste da Bahia, Vitória da Conquista, 2005.

MAGUIRE, J. D. Speed of germination-aid in selection and evaluation for seedling emergence and vigor. Crop Science, v.1, p.176-177, 1962.

MARTINS, C. C.; NAKAGAWA, J.; BOVI, M. L. Efeito da posição da semente no substrato e no crescimento inicial das plântulas de palmitovermelho (Euterpe espiritosantensis Fernandes Palmae). Revista Brasileira de Sementes, v.21, n.1, p.164-173, 1999.

MORAES NETO, S. P. et al. Fertilização de mudas de espécies arbóreas nativas e exóticas. Revista Árvore, v.27, n.2, p.129-137, 2003.

NOVAES, A. B. Avaliação morfofisiológica da qualidade de mudas de Pinus taeda L. produzidas em raiz nua e em diferentes tipos de recipientes. 118f. 1998. Tese (Doutorado em Engenharia Florestal) Universidade Federal do Paraná, Curitiba, 1998.

NOVAES, A. B. et al. Avaliação do potencial de regeneração de raízes de mudas de Pinus taeda l., produzidas em diferentes tipos de recipientes, e o seu desempenho no campo. Revista Árvore, v.26, n.6, p.675-681, 2002.

REIS, A. R. et al. Diagnóstico da exigência do cafeeiro em nitrogênio pela utilização do medidor portátil de clorofila. Bragantia, v.65, n.1, p.163-171, 2006.

REIS, E. R. et al. Período de permanência de mudas de Eucalyptus grandis em viveiro baseado em parâmetros morfológicos. Revista Árvore, v.32, n.5, p.809-814, 2008.
SENA JÚNIOR, D. G. et al. Discriminação entre estágios nutricionais na cultura do trigo com técnicas de visão artificial e medidor portátil de clorofila. Engenharia Agrícola, v.28, n.1, p.187-195, 2008.

SOARES, E. R. et al. Desenvolvimento de mudas de pepino em substratos produzidos com resíduos de algodão e de poda de árvores. In: ENCONTRO NACIONAL SOBRE SUBSTRATOS PARA PLANTAS MATERIAIS REGIONAIS COMO SUBSTRATO, 6., Fortaleza, 2008. Anais... Fortaleza, Embrapa Agroindústria Tropical/ SEBRAE/CE/UFC, 2008.

STURION, J. A; ANTUNES, B. M. A. Produção de mudas de espécies florestais. In: GALVÃO, A. P. M. Reflorestamento de propriedades rurais para fins produtivos e ambientais. Colombo: p.125-150, 2000.

STURION, J. A.; GRAÇÃ, L. R.; ANTUNES, J. B. M. Produção de mudas de espécies de rápido crescimento por pequenos produtores. Circular Técnica 37. Colombo: Embrapa Florestas, 2000. 20p.

TAIZ, L.; ZEIGER, E. Fisiologia vegetal. 4. ed. Trad. SANTARÉM, E. R. Porto Alegre: Artmed, 2009. 820p.

WEBER, O. B. et al. Efeito da bactéria diazotrófica em mudas micropropagadas de abacaxizeiros Cayenne Champac em diferentes substratos. Pesquisa Agropecuária Brasileira, Brasília: v.38, n.6, p.689-696, jun. 2003.

WENDLING, I.; GUASTALA, D.; DOMINGOS, D. M. Substratos para produção de mudas de ervamate em tubetes plásticos. Boletim de Pesquisa Florestal, Colombo: n.52, p.21-36, jan./jun. 2006. 